\title{
Differences in cardiac phenotype and natural history of laminopathies with and without neuromuscular onset
}

\author{
Raffaello Ditaranto ${ }^{1}$, Giuseppe Boriani ${ }^{2}$, Mauro Biffi ${ }^{1}$, Massimiliano Lorenzini ${ }^{1,3}$, Maddalena Graziosi ${ }^{1}$, \\ Matteo Ziacchi ${ }^{1}$, Ferdinando Pasquale ${ }^{1}$, Giovanni Vitale ${ }^{1}$, Alessandra Berardini ${ }^{1}$, Rita Rinaldi ${ }^{4}$, Giovanna Lattanzi ${ }^{5}$, \\ Luciano Potena ${ }^{1}$, Sofia Martin Suarez ${ }^{1}$, Maria Letizia Bacchi Reggiani ${ }^{1}$, Claudio Rapezzi ${ }^{1}$ and Elena Biagini ${ }^{*}$
}

\begin{abstract}
Objective: To investigate differences in cardiac manifestations of patients affected by laminopathy, according to the presence or absence of neuromuscular involvement at presentation.

Methods: We prospectively analyzed 40 consecutive patients with a diagnosis of laminopathy followed at a single centre between 1998 and 2017. Additionally, reports of clinical evaluations and tests prior to referral at our centre were retrospectively evaluated.
\end{abstract}

Results: Clinical onset was cardiac in 26 cases and neuromuscular in 14. Patients with neuromuscular presentation experienced first symptoms earlier in life (11 vs 39 years; $p<0.0001)$ and developed atrial fibrillation/flutter (AF) and required pacemaker implantation at a younger age (28 vs 41 years [ $p=0.013]$ and 30 vs 44 years $[p=0.086$ ] respectively), despite a similar overall prevalence of AF ( $57 \%$ vs $65 \% ; p=0.735)$ and atrio-ventricular ( $A-V$ ) block ( $50 \%$ vs $65 \% ; p=0.500)$. Those with a neuromuscular presentation developed a cardiomyopathy less frequently (43\% vs $73 \% ; p=0.089$ ) and had a lower rate of sustained ventricular tachyarrhythmias ( $7 \%$ vs $23 \% ; p=0.387$ ). In patients with neuromuscular onset rhythm disturbances occurred usually before evidence of cardiomyopathy. Despite these differences, the need for heart transplantation and median age at intervention were similar in the two groups ( $29 \%$ vs $23 \%$ [ $p=0.717$ ] and 43 vs 46 years [ $p=0.593$ ] respectively).

Conclusions: In patients with laminopathy, the type of disease onset was a marker for a different natural history. Specifically, patients with neuromuscular presentation had an earlier cardiac involvement, characterized by a linear and progressive evolution from rhythm disorders (AF and/or A-V block) to cardiomyopathy.

Keywords: Lamin, Emerin, Neuromuscular disorders, Atrial fibrillation, Bradyarrhythmias, Ventricular tachycardias, Familial cardiomyopathies

\section{Introduction}

Laminopathies are a group of inherited conditions due to mutations in the LMNA gene, that encodes the nuclear envelope proteins lamin A and C, via alternate splicing [1]. Laminopathies are characterized by a high phenotypic heterogeneity including heart disease, neuromuscular disorders, premature aging and metabolic disorders [2-5]. $L M N A$ associated cardiac and skeletal muscle disease -

\footnotetext{
*Correspondence: elena.biagini73@gmail.com

${ }^{1}$ Cardiology Unit, Cardio-Thoracic-Vascular Department, Sant'Orsola-Malpighi Hospital, University of Bologna, Via G. Massarenti 9, 40138 Bologna, Italy Full list of author information is available at the end of the article
}

often coexisting in the same patient - are the most frequent clinical manifestations. The spectrum of cardiac involvement ranges from supraventricular tachyarrhythmias and/ or conduction system disease to dilated cardiomyopathy (DCM) and ventricular tachyarrhythmias [6-12]. Sudden cardiac death may occur due to bradyarrhythmias or to malignant ventricular arrhythmias [13], even in the presence of mild left ventricle systolic dysfunction. Similarly, LMNA-related neuromuscular disorders are characterized by a wide heterogeneity in clinical manifestations, Emery Dreifuss muscular dystrophy (EDMD) being the most common phenotype. EDMD is typically characterized by

(c) The Author(s). 2019 Open Access This article is distributed under the terms of the Creative Commons Attribution 4.0 International License (http://creativecommons.org/licenses/by/4.0/), which permits unrestricted use, distribution, and 
early onset joint contractures with slowly progressive scapulo-humero-peroneal muscle weakness, and can be caused by mutations in genes other than $L M N A$, mainly $E M D$ that encodes emerin.

Although clinical manifestation in patients with $L M N A$ mutations have been extensively described, the exact time course of cardiological and neuromuscular disease and their relation remain unclear. Specifically, it is not known if a neuromuscular onset is associated with a different cardiac phenotype or cardiac disease progression. The aim of this study was therefore to investigate differences in cardiac phenotype and natural history in relation to the presence of neuromuscular involvement at presentation, in patients with a diagnosis of laminopathy. Furthermore, in order to test the hypothesis that neuromuscular presentation (phenotype) per se might be associated with a specific cardiac natural history, irrespective of genetics, we compared patients with neuromuscular presentation and LMNA or EMD mutations.

\section{Methods}

In this observational study, we prospectively evaluated all LMNA mutation carriers from a single Italian centre (S.Orsola-Malpighi University Hospital, Bologna), between December 1998 and November 2017. We also retrospectively examined clinical, ECG and echocardiographic reports available prior to evaluation at our centre. According to the presence of signs and/or symptoms of skeletal myopathy at clinical presentation (not necessarily in our centre), patients were divided into two groups: "with neuromuscular onset" and "without neuromuscular onset". Data of 7 patients with $E M D$-related disease were also recorded. All patients underwent periodical clinical, electrocardiographic and echocardiographic monitoring.

DCM was defined as the presence of left ventricular (LV) dilation and systolic dysfunction in the absence of abnormal loading conditions (hypertension or valve disease) or coronary artery disease sufficient to cause global systolic impairment [14]. "Hypokinetic non-dilated cardiomyopathy" (HNDC) was defined as LV ejection fraction (EF) $<45 \%$ or biventricular global systolic dysfunction in absence of dilatation [15]. Restrictive cardiomyopathy was defined as a nondilated LV with normal wall thickness and EF, and severe diastolic dysfunction with restrictive filling pattern, elevated filling pressures and dilated atria [14]. Sustained ventricular tachyarrhythmias (SVT) were defined as ventricular tachyarrhythmias with a rate $\geq 120 / \mathrm{min}$, lasting $>30 \mathrm{~s}$.

A neurological involvement was investigated on the basis of personal history and/or symptoms including joint contractures, muscle weakness or wasting, orthopaedic surgery, and exercise tolerance. Electromyography (EMG), muscle imaging or muscle biopsy were performed in selected cases. Patients underwent periodical neurological evaluation, even when no skeletal muscle involvement was recorded at first evaluation. Elevation of serum creatine kinase $(\mathrm{CK})$ in isolation was not considered diagnostic of neuromuscular involvement in absence of clinical, EMG, imaging or histological evidence of skeletal myopathy.

A detailed family history was collected for each proband in order to identify other potentially affected family members. A genetic diagnosis was made by DNA sequencing from peripheral blood and mutations were considered pathogenic if previously described in literature, in the presence of co-segregation or based on the site and the type of mutation. After mutation identification, cascade genetic screening was performed in family members, following informed consent.

Continuous data distribution was assessed with the Shapiro-Wilk test and expressed as median and interquartile range (IQR). Data were compared by the Fisher's exact test for proportions, and Mann Whitney test for continuous variables. Clinical events were reported as counts and percentages (i.e. events/total number of patients $\times 100$ ). Data were collected following informed consent.

\section{Results}

Of the 41 LMNA mutation carriers, 40 were clinically affected. Table 1 reports clinical, ECG and echocardiographic characteristics at first evaluation at our centre. Fourteen patients were assigned to the group "with neuromuscular onset" and 26 to the group "without neuromuscular onset". A single $L M N A$ mutation carrier, who did not have any cardiac or neuromuscular phenotypic expression at baseline or during follow up, was excluded from the analysis.

\section{Patients with neuromuscular onset}

Among the 14 patients in this group males and females were equally represented. LMNA mutations were: 12 missense, 1 splice site, and 1 deletion (Table 2). Median age at first evaluation at our Centre was 31 years (IQR 20-44). The diagnosis of laminopathy occurred following familial screening in 2 patients. At time of genetic diagnosis, overt neuromuscular involvement was present in all cases whereas none had cardiac involvement. Eleven patients (79\%) were diagnosed with EDMD in the first or second decade of life and $3(21 \%)$ were diagnosed with a non-specific myopathy in the third decade; median age at diagnosis was 11 years (IQR $8-30$ ).

Nine patients (64\%) developed cardiac involvement prior to referral to our centre (Fig. 1a summarizes the spectrum of cardiac phenotypic expression). All had rhythm disturbances: 2 (14\%) had atrial fibrillation (AF), 1 (7\%) atrioventricular (A-V) block, 1 (7\%) sinus node dysfunction, and 5 (36\%) had a combination of brady- and atrial tachyarrhythmias (Fig. 2). Cardiomyopathy was also present in 5 patients (36\%): 2 DCM, 2 HNDC and 1 isolated right ventricular cardiomyopathy mimicking arrhythmogenic 
Table 1 Characteristics at first clinical evaluation at our centre of patients with LMNA mutations with and without neuromuscular onset

\begin{tabular}{|c|c|c|c|c|}
\hline & Overall & With neurological Onset & Without neurological onset & $P$ value \\
\hline Number of patients & 40 & $14(35)$ & $26(65)$ & \\
\hline Number of families & 31 & $12(39)$ & $19(61)$ & \\
\hline Males & $22(55)$ & $7(50)$ & $15(58)$ & 0.744 \\
\hline Age at symptom onset, yrs & $33(21-42)$ & $11(8-30)$ & $39(32-46)$ & $<0.0001$ \\
\hline Age at first contact at our center, yrs & $39(29-74)$ & $31(20-44)$ & $43(36-49)$ & \\
\hline Follow-up duration, months & $30(6-70)$ & $24(12-101)$ & $32(8-63)$ & \\
\hline \multicolumn{5}{|l|}{ Diagnostic pathway } \\
\hline Signs or symptoms & & $12(86)$ & $19(73)$ & 0.452 \\
\hline Screening & & $2(14)$ & $7(27)$ & \\
\hline Cardiomyopathy & $24(60)$ & $5(36)$ & $19(73)$ & 0.040 \\
\hline Dilated CMP & $13(32)$ & $2(14)$ & $11(42)$ & 0.089 \\
\hline Hypokinetic non dilated CMP & $8(20)$ & $2(14)$ & $6(23)$ & 0.688 \\
\hline Restrictive CMP & $2(5)$ & 0 & $2(8)$ & 0.533 \\
\hline Right ventricle CMP & $1(2)$ & $1(7)$ & 0 & 0.350 \\
\hline History of atrial fibrillation & $17(42)$ & $6(43)$ & $11(42)$ & 1.000 \\
\hline Sinus node dysfunction & $4(10)$ & $3(21)$ & $1(4)$ & 0.114 \\
\hline Atrio-ventricular block & $23(57)$ & $6(43)$ & $17(65)$ & 0.197 \\
\hline 1st degree & $8(20)$ & $2(14)$ & $6(23)$ & \\
\hline 2nd degree & $7(17)$ & $2(14)$ & $5(19)$ & \\
\hline 3rd degree/high degree & $8(20)$ & $2(14)$ & $6(23)$ & \\
\hline \multicolumn{5}{|l|}{ Positive familial history for } \\
\hline Sudden death & $7(17)$ & $3(21)$ & $4(15)$ & 0.678 \\
\hline PM implantation (high degree AVB) & $11(27)$ & $3(21)$ & $8(30)$ & 0.715 \\
\hline CMP & $16(40)$ & $5(36)$ & $11(42)$ & 0.746 \\
\hline NYHA class III- IV & $7(17)$ & $4(28)$ & $3(12)$ & 0.214 \\
\hline PM recipients & $8(20)$ & $2(14)$ & $6(23)$ & 0.688 \\
\hline ICD recipients & $8(20)$ & $1(7)$ & $7(27)$ & 0.221 \\
\hline
\end{tabular}

Values are expressed as $\mathrm{N}, \mathrm{N}$ (\%) or median (interquartile range)

$L M N A, E M D$ gene codifying for lamin A/C and emerin, respectively, ICD implantable cardioverter defibrillator, $P M$ pacemaker, $A V B$ atrio-ventricular block, NYHA New York Heart Association

right ventricular cardiomyopathy. Patients with LV cardiomyopathy had a severe LV dysfunction (LVEF $\leq 35 \%)$ and 3 had biventricular involvement.

Two patients (14\%) had previously undergone pacemaker (PM) implantation for A-V block, while a single patient had a primary prevention implantable cardiac resynchronization therapy defibrillator (CRT-D). Serum CK levels were raised in $65 \%$ of the patients, with a mean abnormal value of $631 \mathrm{UI} / \mathrm{L}$.

\section{Patients without neuromuscular onset}

Fifteen (58\%) of the 26 patients were males, median age at first evaluation at our centre was 43 years (IQR 36-49). Diagnosis was made due to rhythm disturbances or heart failure symptoms in most cases $(n=19,73 \%)$. Seven patients $(27 \%)$ were identified after family screening. LMNA mutations were: 16 missense, 7 splice site, 2 deletion and 1 frameshift (Table 3).

At first clinical evaluation at our centre, 19 patients (73\%) had a cardiomyopathy, in isolation $(n=3,11 \%)$ or associated with arrhythmias (AF $n=5,19 \%$; A-V block $n=6$, 23\%; both $n=5$, 19\%; Fig. 3). Seven patients (27\%) had arrhythmias in absence of cardiomyopathy: 1 (4\%) AF, 5 (19\%) A-V block and 1 (4\%) a combination of sinus node and A-V node dysfunction. Overall, AF was present in 11 patients $(42 \%)$. Figure $1 \mathrm{~b}$ summarizes the spectrum of cardiac phenotypic expression. Six patients (23\%) had previously undergone PM implantation for A-V block and 7 patients $(27 \%)$ had received a primary prevention implantable cardioverter defibrillator (ICD).

Cardiomyopathy phenotype included: 11 DCM, 6 HNDC and 2 restrictive cardiomyopathy. Among the 
Table 2 Genetics of LMNA mutated patients with neurological onset $(N=14)$

\begin{tabular}{|c|c|c|c|c|c|}
\hline & Gene & Location & Nucleotide Change & Protein Change & Predicted Effect \\
\hline \multicolumn{6}{|l|}{ Family 1} \\
\hline F; 16 yo & LMNA & Exon 4 & c.746G > A & p.Arg249GIn & Missense \\
\hline \multicolumn{6}{|l|}{ Family 2} \\
\hline F; 50 yo & LMNA & Exon 9 & C.1580G > C & p.Arg.527.Pro & Missense \\
\hline \multicolumn{6}{|l|}{ Family 3} \\
\hline M; 38 yo & LMNA & Exon 11 & c.1930C > T & p.Arg644Cys & Missense \\
\hline M; 38 yo & LMNA & Exon 11 & c.1930C > T & p.Arg644Cys & Missense \\
\hline \multicolumn{6}{|l|}{ Family 4} \\
\hline F; 46 yo & LMNA & $\begin{array}{l}\text { Exon } 3 \\
\text { Exon } 4\end{array}$ & $\begin{array}{l}\text { c. } 569 \mathrm{G}>\mathrm{A}_{i} \\
\text { c. } 746 \mathrm{G}>\mathrm{A}\end{array}$ & $\begin{array}{l}\text { p.Arg190Gln } \\
\text { p.Arg249GIn }\end{array}$ & $\begin{array}{l}\text { Missense } \\
\text { Missense }\end{array}$ \\
\hline \multicolumn{6}{|l|}{ Family 5} \\
\hline F; 34 yo & LMNA & Exon 1 & c.203_208 (delAGGTGG) & p.Glu68_Val69 del & Deletion \\
\hline \multicolumn{6}{|l|}{ Family 6} \\
\hline M; 52 yo & LMNA & Exon 4 & c.746G > A & p.Arg249Gln & Missense \\
\hline \multicolumn{6}{|l|}{ Family 7} \\
\hline M; 46 yo & LMNA & Exon 9 & c.1567G > A & p.Gly523Arg & Missense \\
\hline \multicolumn{6}{|l|}{ Family 8} \\
\hline M; 17 yo & LMNA & Exon 4 & c.775 T > G & p.Tyr259Asp & Missense \\
\hline \multicolumn{6}{|l|}{ Family 9} \\
\hline M; 19 yo & LMNA & Exon 4 & c.746 G > A & p.Arg249GIn & Missense \\
\hline \multicolumn{6}{|l|}{ Family 10} \\
\hline F; 29 yo & LMNA & Intron 9 & c. $1608+1 G>T$ & - & Splice site \\
\hline \multicolumn{6}{|l|}{ Family 11} \\
\hline F; 22 yo & LMNA & Exon 1 & c. $188 \mathrm{~T}>\mathrm{A}$ & p.lle63Asn & Missense \\
\hline F; 19 yo & LMNA & Exon 1 & c.188T > A & p.lle63Asn & Missense \\
\hline \multicolumn{6}{|c|}{ Family 12} \\
\hline M; 27 yo & LMNA & Exon 4 & c.746G > A & p.Arg249Gln & Missense \\
\hline
\end{tabular}

$M$ male, $F$ female, yo years old. The age reported refers to first contact at our centre

17 patients with LV systolic dysfunction, 7 (41\%) had a severe impairment (LVEF $\leq 35 \%), 6$ (35\%) had biventricular involvement and 3 (17\%) had increased LV trabeculations. One 54-year old patient with a history of complete A-V block and AF had a biventricular cardiomyopathy with multiple aneurysms in the diaphragmatic and free wall of the right ventricle. Coronary arteries were unobstructed at angiography. Cardiac magnetic resonance showed a severely dilated left ventricle (indexed end diastolic volume: $146 \mathrm{~mL} / \mathrm{m} 2$ ) with systolic dysfunction (EF $40 \%$ ) and mildly dilated right ventricle (indexed end diastolic volume: $111 \mathrm{ml} / \mathrm{m} 2$ ) with reduced $\mathrm{EF}(40 \%)$ and confirmed the wall motion abnormalities (Fig. 4 a-b). Tissue characterization (Fig. $4 \mathrm{c}$-d) revealed multiple areas of fibro-fatty replacement. In this case, possible phenocopies including desmosomal-related cardiomyopathy and sarcoidosis were excluded by genetic analysis, positron emission tomography, lung $\mathrm{CT}$ and, endomyocardial biopsy.
No patient had evidence of neuromuscular involvement. Serum CK levels were raised in $47 \%$ of the patients with a mean abnormal value of $217 \mathrm{UI} / \mathrm{L}$.

\section{Follow-up of patients with neuromuscular onset}

Median follow up was 24 months (IQR 12-101). New onset AF was recorded in 2 patients, therefore $57 \%$ experienced atrial tachyarrhythmias by the end of the follow-up. In one patient, the atrial conduction disease progressed to atrial paralysis. Three patients underwent PM implantation for A-V block $(n=1)$, sinus node disease $(n=1)$ or both $(n=$ 1). A primary prevention CRT-D was implanted in a patient with new onset HNDC due to positive family history for sudden death, high inducibility of VT on electrophysiological study and moderate LV dysfunction. The patient affected by right ventricular cardiomyopathy received a primary prevention ICD, due to severe right ventricular dysfunction, non-sustained ventricular tachycardias and the need of a pacing for sinus and $\mathrm{A}-\mathrm{V}$ node dysfunction. 


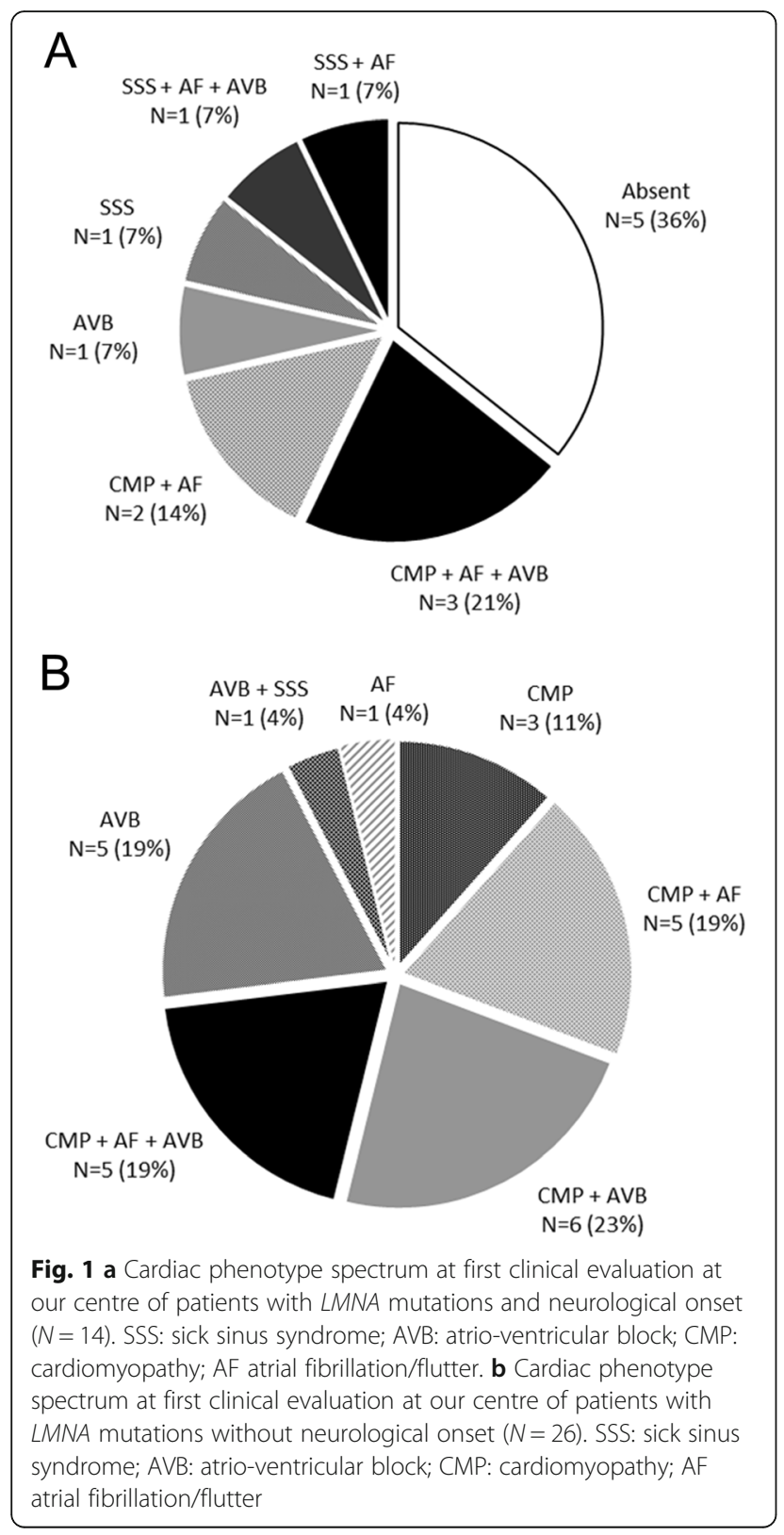

Thereafter he experienced an appropriate ICD activation and a progression towards severe biventricular involvement. No sudden death occurred. Five patients with cardiomyopathy had hospital admissions due to heart failure during follow-up and 4 of them subsequently underwent heart transplantation (median age 43 [IQR 34-48]).

\section{Follow-up of patients without neuromuscular onset}

New onset AF was reported in 6 patients (23\%) during a median follow up of 32 months (IQR 8-63); 65\% of patients had atrial tachyarrhythmias at the end of follow up. With the exception of 2 patients with atrial flutter who were treated successfully with cavo-tricuspid isthmus ablation - the attempts of rhythm control with electrical or pharmacological cardioversion were ineffective. No patients underwent pulmonary vein isolation. Atrial paralysis was documented in a single patient. One patient underwent PM implantation due to A-V block. A primary prevention ICD was implanted in 7 patients (4 new implants and 3 device upgrades) and 1 ICD was implanted for secondary prevention. Four of the ICD recipients (50\%) received a CRT-D device. During follow up 6 patients (23\%) experienced appropriate shocks and/or antitachycardia pacing for ventricular arrhythmias, with an arrhythmic storm in 3 cases. Six (23\%) patients underwent cardiac transplantation (median age 46 [IQR 34-53]) due to end stage heart failure (5/6) or to recurrent ventricular arrhythmias (1/6). One patient developed a mild neuromuscular involvement, with muscle atrophy involving the shoulder girdle. Table 4 compares clinical events reported during follow-up in the two groups.

\section{Differences in clinical manifestations between patients with and without neuromuscular onset}

Patients with neuromuscular onset had an earlier presentation, during infancy or adolescence in most of the cases (median age 11 years), mainly as EDMD, followed by the first evidence of cardiac disease by a median age of 13 years (IQR 10-15) (maximum timelag 38 years). In patients without neuromuscular onset, first cardiac symptoms occurred later in life, at a median age of 39

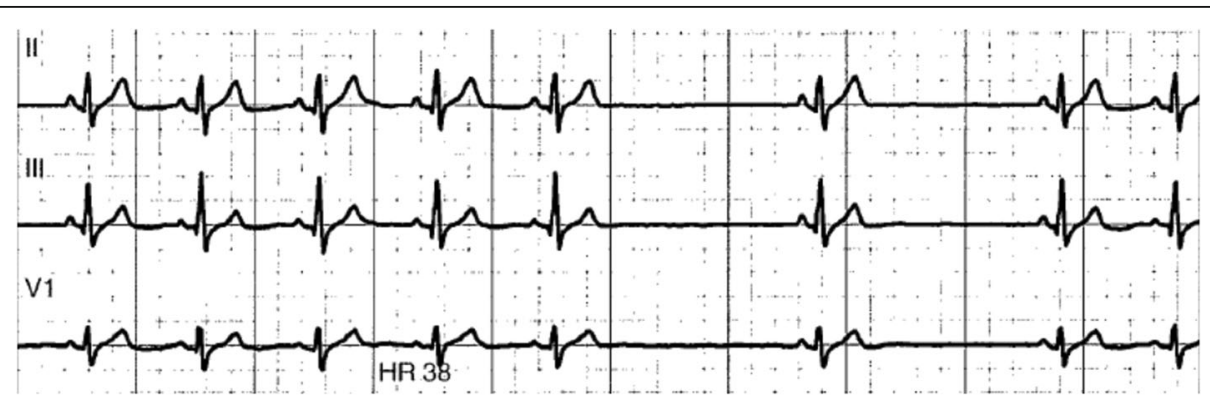

Fig. 2 Second degree sino-atrial block with 2:1 conduction ratio in a 22-year old female, with Emery Dreifuss muscular dystrophy due to p.lle63Asn missense LMNA mutation 
Table 3 Genetics and cardiac manifestations of LMNA mutated patients without neurological onset $(N=26)$

\begin{tabular}{|c|c|c|c|c|c|}
\hline & Gene & Location & Nucleotide Change & Protein Change & Predicted Effect \\
\hline \multicolumn{6}{|l|}{ Family 1} \\
\hline M; 53 уо & LMNA & Exon 6 & c. $1004 \mathrm{G}>\mathrm{A}$ & p.Arg335Gln & Missense \\
\hline M; 26 yo & LMNA & Exon 6 & c. $1004 \mathrm{G}>\mathrm{A}$ & p.Arg335Gln & Missense \\
\hline \multicolumn{6}{|l|}{ Family 2} \\
\hline $\mathrm{F} ; 47$ yo & LMNA & Exon 7 & 1370delA & p.Lys457SerfsX2 & Deletion \\
\hline \multicolumn{6}{|l|}{ Family 3} \\
\hline M; 19 yo & LMNA & Exon 6 & c. $1003 \mathrm{G}>\mathrm{A}$ & p.Arg335Glu & Missense \\
\hline \multicolumn{6}{|l|}{ Family 4} \\
\hline F; 55 yo & LMNA & Exon 11 & c. $1912 \mathrm{G}>\mathrm{A}$ & p.Gly638Arg & Missense \\
\hline \multicolumn{6}{|l|}{ Family 5} \\
\hline M; 54 yo & LMNA & Exon 7 & c. $1202 \mathrm{G}>\mathrm{A}$ & p.Arg $401 \mathrm{His}$ & Missense \\
\hline \multicolumn{6}{|l|}{ Family 6} \\
\hline M; 42 yo & LMNA & Exon 1 & $\mathrm{n} / \mathrm{a}$ & p.Arg72Alafs*24 & Frame shift \\
\hline \multicolumn{6}{|l|}{ Family 7} \\
\hline F; 51 yo & LMNA & Exon 4 & c. $752 \mathrm{~A}>\mathrm{C}$ & p.Gln251Pro & Missense \\
\hline \multicolumn{6}{|l|}{ Family 8} \\
\hline M; 46 yo & LMNA & Exon 9 & C.1517 A > C & p.His506Pro & Missense \\
\hline \multicolumn{6}{|l|}{ Family 9} \\
\hline F; 38 yo & LMNA & Intron 9 & $\mathrm{c} 1608+1 \mathrm{G}>\mathrm{T}$ & - & Splice site \\
\hline M; 35 yo & LMNA & Intron 9 & $\mathrm{c} 1608+1 \mathrm{G}>\mathrm{T}$ & - & Splice site \\
\hline \multicolumn{6}{|l|}{ Family 10} \\
\hline $\mathrm{F} ; 29$ yo & LMNA & Exon 3 & c.548 T >C & p.Leu183Pro & Missense \\
\hline \multicolumn{6}{|c|}{ Family 11} \\
\hline F; 41 yo & LMNA & Exon 6 & c. $1007 \mathrm{G}>\mathrm{A}$ & p.Arg336Gln & Missense \\
\hline \multicolumn{6}{|l|}{ Family 12} \\
\hline$M ; 60$ yo & LMNA & Intron 1 & $\begin{array}{l}\text { c. } 357-1 G>A \\
\text { IVS1-1G > A }\end{array}$ & - & Splice site \\
\hline $\mathrm{F} ; 46$ yo & LMNA & Intron 1 & $\begin{array}{l}\text { C. } 357-1 G>A \\
\text { IVS1-1G > A }\end{array}$ & - & Splice site \\
\hline$F ; 49$ yo & LMNA & Intron 1 & $\begin{array}{l}\text { C. } 357-1 G>A \\
\text { IVS1-1G > A }\end{array}$ & - & Splice site \\
\hline$F ; 49$ yo & LMNA & Intron 1 & $\begin{array}{l}\text { C. } 357-1 G>A \\
\text { IVS1-1G >A }\end{array}$ & - & Splice site \\
\hline M; 21 yo & LMNA & Intron 1 & $\begin{array}{l}\text { C. } 357-1 G>A \\
\text { IVS1-1G >A }\end{array}$ & - & Splice site \\
\hline \multicolumn{6}{|c|}{ Family 13} \\
\hline M; 38 yo & LMNA & Exon 6 & C. $1129 C>T$ & p.Arg377Cys & Missense \\
\hline \multicolumn{6}{|l|}{ Family 14} \\
\hline M; 50 уо & LMNA & Exon 2 & $c .481 G>A$ & p.Glu161Lys & Missense \\
\hline \multicolumn{6}{|l|}{ Family 15} \\
\hline M; 46 yo & LMNA & Exon 2 & c. $466 C>$ A & p.Arg156Ser & Missense \\
\hline M; 34 yo & LMNA & Exon 2 & c. $466 C>$ C & p.Arg156Ser & Missense \\
\hline \multicolumn{6}{|l|}{ Family 16} \\
\hline $\mathrm{M} ; 44$ yo & LMNA & Exon 4 & c. 671 C > T & p.Thr224lle & Missense \\
\hline \multicolumn{6}{|c|}{ Family 17} \\
\hline F; 39 yo & LMNA & Exon 2 & $c .481 G>A$ & p.Glu161Lys & Missense \\
\hline
\end{tabular}


Table 3 Genetics and cardiac manifestations of LMNA mutated patients without neurological onset $(N=26)(C o n t i n u e d)$

\begin{tabular}{llllll}
\hline & Gene & Location & Nucleotide Change & Protein Change & Predicted Effect \\
\hline Family $\mathbf{1 8}$ & & & & & MLys171Asn \\
F; 37 yo & LMNA & Exon 2 & c.513G > A & & Missense \\
Family $\mathbf{1 9}$ & & & p.Ala287LeufsX191 & Deletion \\
F; 36 yo & LMNA & Exon 5 & c.855delG & \\
\hline
\end{tabular}

$M$ male, $F$ female, yo years old. The age reported refers to first contact at our centre. $n / a$ not available

years $(p<0.0001)$. Regarding arrhythmias, at the end of the follow-up A-V block (of any degree) and AF had a similar prevalence between the two groups (50\% vs $65 \%$, $p=0.500$ and $57 \%$ vs $65 \% ; p=0.735$ respectively). Sinus node dysfunction was more frequent in patients with skeletal myopathy ( $21 \%$ vs $4 \%$; $p=0.114)$, whereas atrial paralysis was reported in one patient for each group. Patients with neuromuscular presentation (Fig. 5) experienced earlier AF (age 28 vs 41, $p=0.013$ ) and PM implantation (age 30 vs $44 ; p=0.086$ ). The percentage of patients requiring permanent pacing (including PM recipients and those who received an ICD due to a concomitant indication for prevention of ventricular arrhythmias) was equal in the two groups ( $42 \%$ vs $42 \%$; $p=1.000$ ).

Patients without neuromuscular presentation had a higher prevalence of cardiomyopathy ( $73 \%$ vs $43 \%, p=$ 0.089 ) and were older at diagnosis (age 42 vs $35, p=0.259$ ). DCM was the dominant phenotype in this group (58\% of all cardiomyopathies), whereas DCM and HNDC where equally represented in the other group. The higher prevalence of heart muscle involvement in patients without neuromuscular onset was associated with a higher number of implanted ICDs $(58 \%$ vs $21 \%, p=0.045)$ and a higher burden of SVT ( $23 \%$ vs $7 \%, p=0.387$ ). Despite this, no significant differences were reported in the prevalence of heart transplantation ( $23 \%$ vs $29 \% ; p=0.717)$ or in the median recipient age (43 vs $46 ; p=0.593$ ).

All patients with neuromuscular presentation who received a diagnosis of cardiomyopathy had a previous history of rhythm disturbance with the exception of 2 cases, where the diagnosis was concomitant. On the contrary, no pattern of progression from rhythm disturbance to cardiomyopathy was present in those without a neuromuscular presentations: $\mathrm{AF}$ and $\mathrm{A}-\mathrm{V}$ block could precede the diagnosis of cardiomyopathy, be diagnosed at the same time or later. Figure 6 shows the different
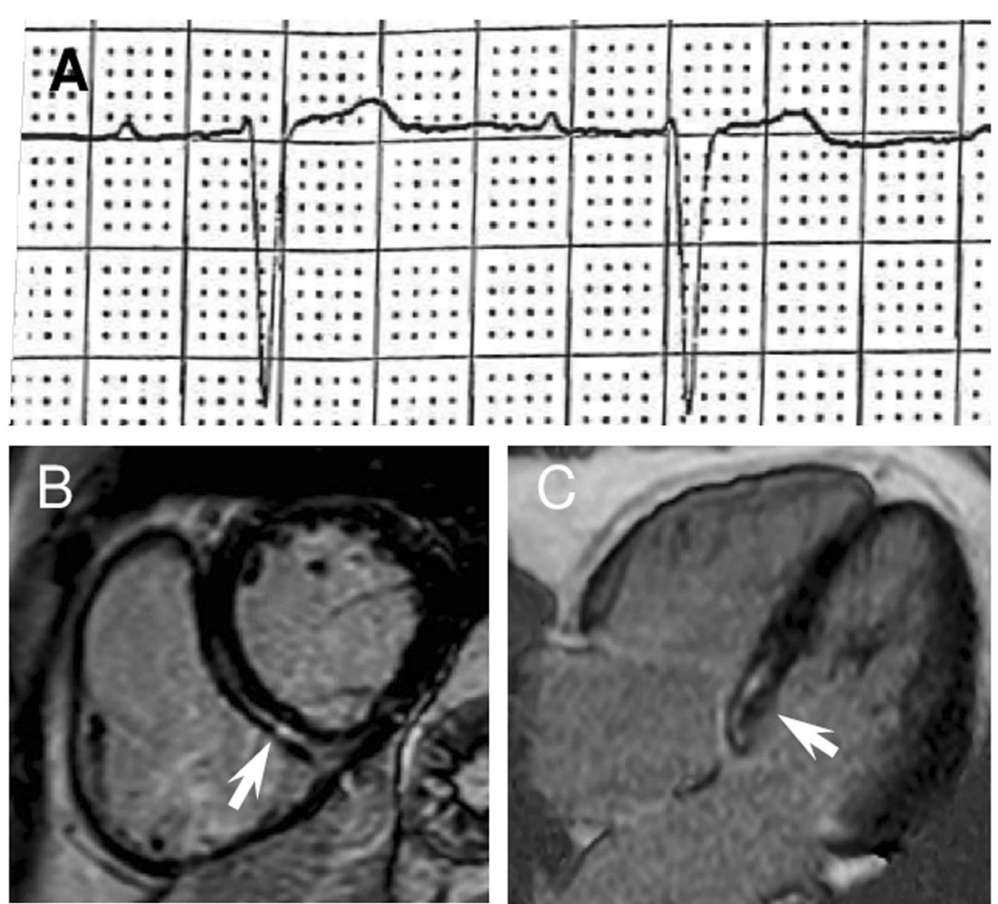

Fig. 3 43-year old male with a LMNA frameshift mutation without neuromuscular presentation. a V1 lead ECG showing first degree atrioventricular block, b-c cardiac magnetic resonance showing midwall late gadolinium enhancement in the basal interventricular septum. Suggestive for myocardial fibrosis 

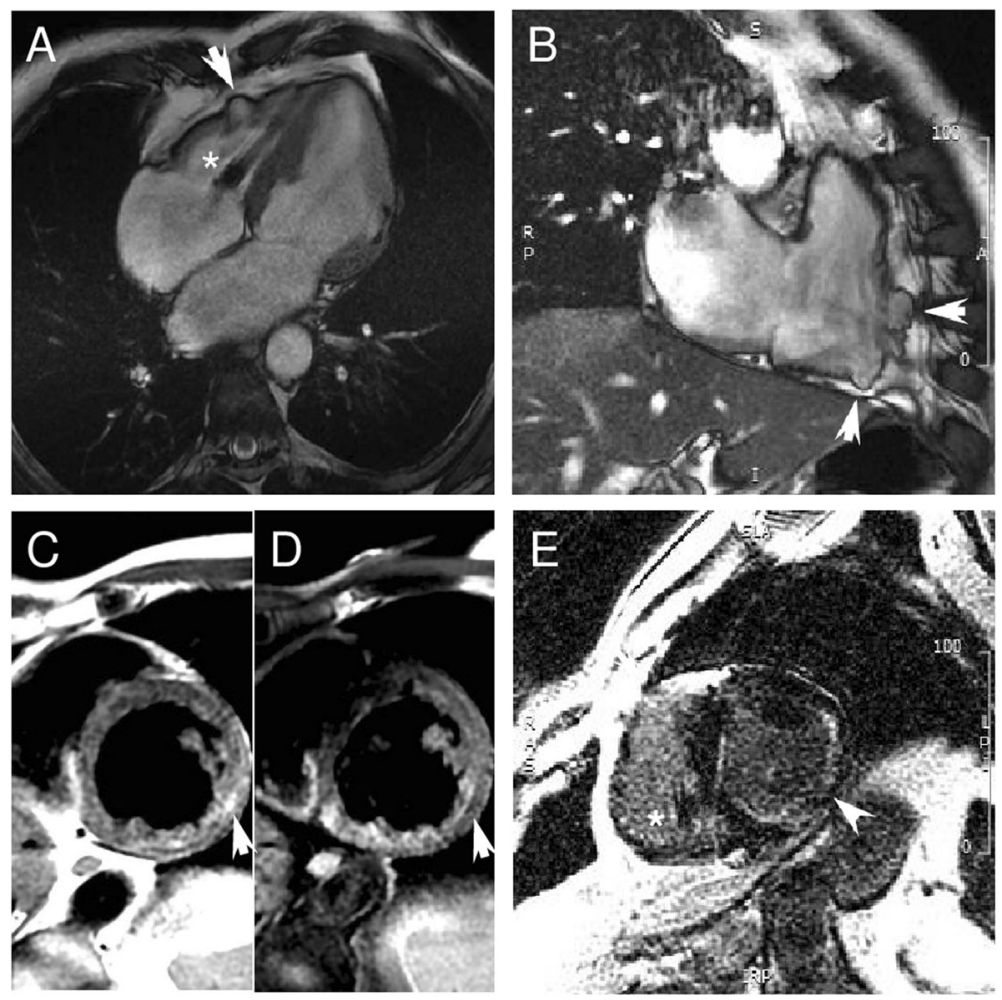

Fig. 4 CMR of a 54-year male, carrier of p.Arg401His missense LMNA mutation, affected by DCM. (a-b) Four chamber and RV long axis SSFP images show biventricular dilation, bulging of the RV free wall (white arrow, panel a-b) and diaphragmatic wall (white arrow, panel b). (c) Two chamber T1-weighted and (d) fat suppressed T1-weighted slices showing LV fatty replacement of mid lateral wall (white arrow). (e) IR LGE slice showing fibrosis in the infero-lateral wall (with focal transmural pattern) and in the interventricular septum (image quality was due to respiratory artifacts and to the presence of pacemaker $\left.{ }^{*}\right]$ ). CMR: cardiac magnetic resonance. DCM: dilated cardiomyopathy. SSFP: steady-state free precession. LV-RV: left-right ventricle. IR LGE: inversion recovery late gadolinium enhancement

overall prevalence of clinical events between the two populations.

\section{Clinical characteristics and follow up of patients with emerinopathy}

Seven male patients ( 4 families) affected by X-linked EDMD were referred to our Centre at a median age of 26 years (IQR 14-30) and followed for a median time of 108 months (IQR 72-172). All had neuromuscular symptoms as first evaluation, with a median age of 6 years (IQR 5-8). At last follow up 6 patients (85\%) had cardiac involvement. All developed AF at a median age of 27 [IQR 23-37]) and 5 required PM implantation at a median age of 23 [IQR 22-24] due to A-V block $(n=1)$, sinus node dysfunction $(n=1)$ or both $(n=3)$. A single patient developed cardiomyopathy with mild systolic dysfunction; none had ventricular arrhythmias. The time interval from neurological to cardiac disease onset was of 14,5 years (IQR 14-15). Compared to patients with LMNA mutations and a neurological onset, patients with emerinopathy presented a higher burden of AF $(85 \%$ vs $57 \% ; p=0.337)$ that occurred an earlier age (27 vs 31

Table 4 Clinical events reported during follow-up in LMNA mutated patients

\begin{tabular}{llll}
\hline & Patients with neurological onset $(N=14)$ & Patients without neurological onset $(N=26)$ & $P$ value \\
\hline New onset of atrial fibrillation (any form) & $2(14)$ & $6(23)$ & 0.688 \\
PM implantation & $3(21)$ & $1(4)$ & 0.114 \\
ICD implantation & $2(14)$ & $8(31)$ & $6(23)$ \\
SVT/arrhythmic storm & $1(7)$ & $11(42)$ & $6(23)$ \\
Admission for heart failure & $5(36)$ & 0 & 0.345 \\
Heart transplantation & $4(29)$ & 0 & 0.717 \\
Thromboembolic events & &
\end{tabular}

Values are expressed as $\mathrm{N}$ or $\mathrm{N}(\%)$. SVT sustained ventricular tachyarrhythmia, $P M$ pacemaker, ICD implantable cardioverter defibrillator 


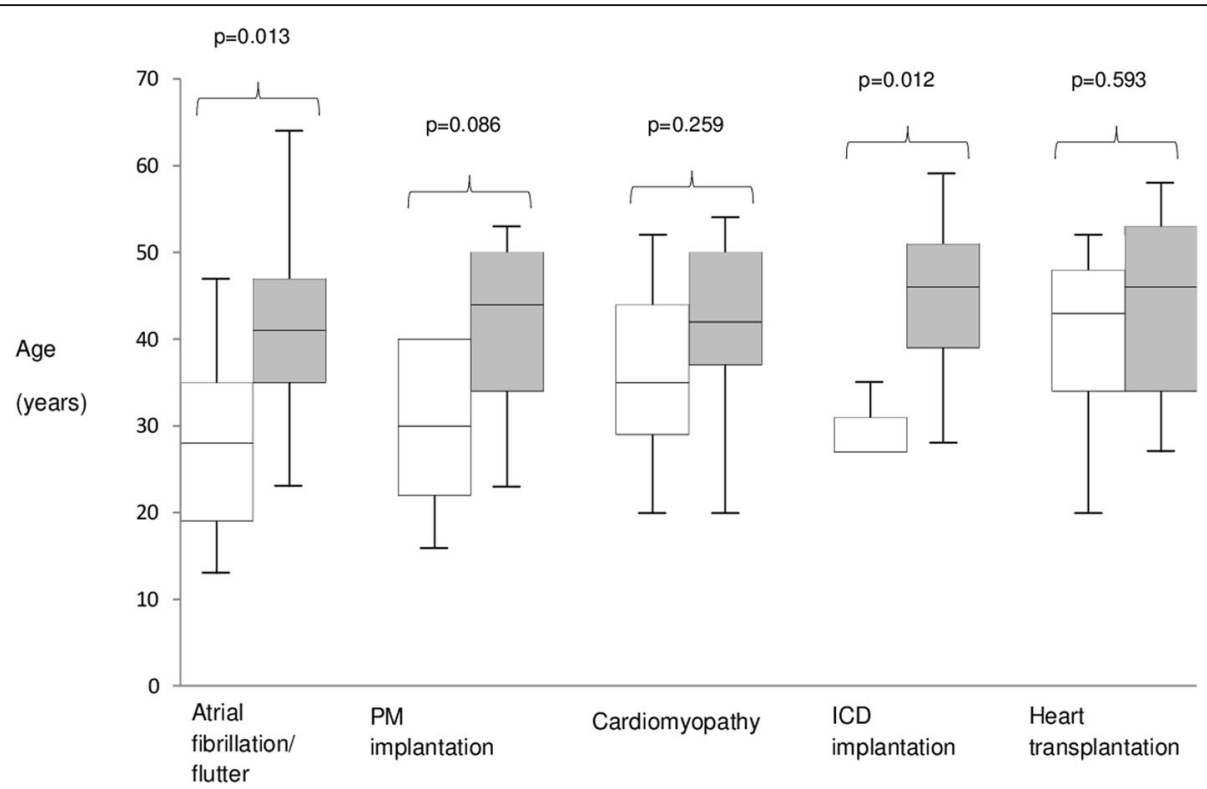

Fig. 5 Box and whiskers plot showing age distribution of different clinical events in LMNA patients with (white) and without (gray) neuromuscular onset. Middle horizontal line inside box indicates median. Bottom and top of the box are 25th and 75th percentiles, the whiskers indicate the lowest and highest value. PM: pacemaker. ICD: implantable cardioverter defibrillator

$p=0.746)$, and a higher rate of PM implantation (71 vs $36 \% ; p=0.182)$ at an earlier age (23 vs $28 ; p=0.461)$. Differently, heart muscle involvement was rare (a single case of cardiomyopathy) and no SVT was documented.

\section{Discussion}

The main findings of our study are: 1) neuromuscular onset is a marker for a specific natural history in laminopathy patients. Specifically, these patients have a linear and predictable progression over time, from muscular dystrophy to rhythm disturbances and finally cardiomyopathy. 2) With the exception of sinus node dysfunction, that was more frequent in EDMD patients, the prevalence of A-V block and AF was similar between the two groups, but patients with a neuromuscular presentation had earlier arrhythmias. 3) Prevalence of cardiomyopathy (particularly DCM) and SVT was higher among patients without neuromuscular onset, although the two groups had a similar rate and age of cardiac transplantation.

Most of the patients with neuromuscular presentation (64\%) developed cardiac involvement later in life: the delay between neuromuscular and cardiac symptom onset was variable and sometimes very long. These findings suggest that serial reassessment of cardiac status in patients with a diagnosis of EDMD is mandatory. On the other hand, patients without neuromuscular onset did not develop an overt skeletal myopathy (with a single exception). The possibility that these patients could develop neuromuscular

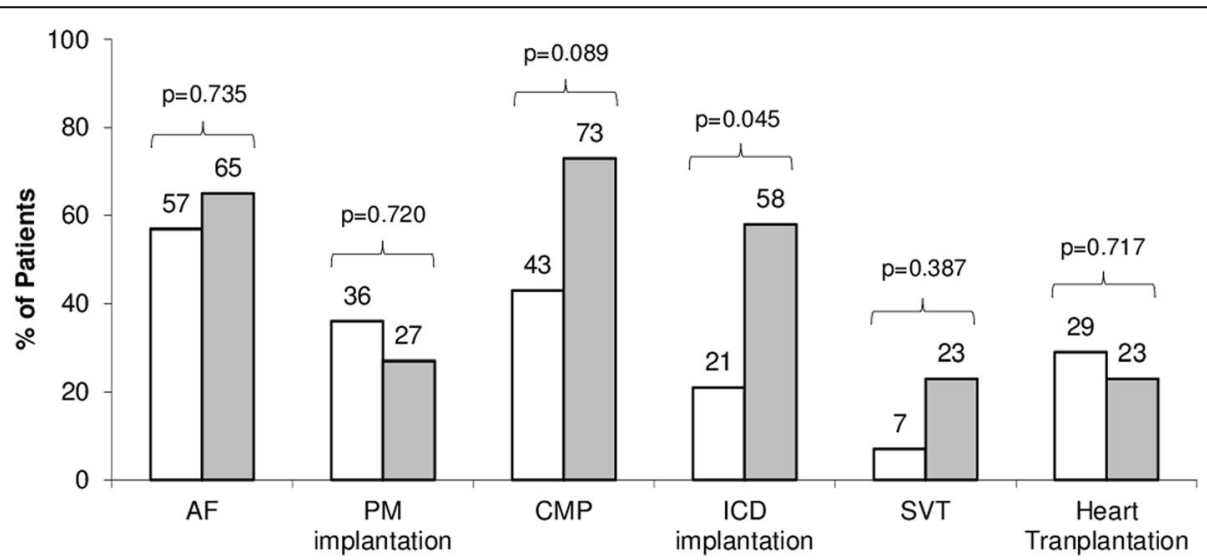

Fig. 6 Different overall prevalence at the end of follow-up of clinical events in LMNA patients with (white) and without (gray) neuromuscular presentation. AF: atrial fibrillation; PM: pacemaker; CMP: cardiomyopathy; SVT: sustained ventricular tachycardias 
involvement in the future cannot however be entirely ruled out due to the limited observation time. Our results are in line with the phenotypic clustering reported by Benedetti et al. [16] in a cohort of patients with $L M N A$ mutations, where those with childhood onset had an almost exclusively skeletal muscle involvement (predominantly EDMD), while patients with adult onset developed cardiac disorders or muscle weakness with a limb-girdle distribution. With the limitation of the small size of the screened families in our study population and the limited numbers of relatives who carried the mutation, all the affected relatives of probands with neuromuscular presentation had a skeletal muscle involvement as clinical onset. At the same time, all the affected relatives of patients with an exclusive cardiac phenotype had an isolated cardiological involvement. Differently from our findings, Bonne et al. and Brodsky et al. $[17,18]$ have previously described the possibility of the coexistence of both phenotypes as clinical onset within the same family.

Our data confirm the high frequency of AF in laminopathies, as well as advanced A-V block, requiring PM implantation at a young age. Although the prevalence of high degree $\mathrm{A}-\mathrm{V}$ block and $\mathrm{AF}$ was similar, irrespective of clinical presentation, patients with neuromuscular onset experienced arrhythmia earlier in life (on average AF and PM implantation occurred more than 10 years earlier). On the other hand, sinus node dysfunction was more frequent in patients with EDMD (21\% vs $4 \%)$. Regarding heart muscle involvement, patients without neuromuscular onset had a prevalence of cardiomyopathy that was almost twice that of the other group (42\% vs $73 \% ; p=0.089$ ), mostly DCM. On the contrary DCM and HNDC were equally distributed in patients with neuromuscular presentation. A progression from HNDC to DCM was not observed in this study, suggesting that they could be the expression of two different pathophysiologic models; however, a limited follow-up duration (median 41 months) and heart failure therapy could have masked this progression. We described two cases, 1 in each group, with a cardiac phenotype that mimicked arrhythmogenic cardiomyopathy. LMNA carriers have been described with clinical, morphological and histological phenotypes overlapping with arrhythmogenic cardiomyopathy [19], in the absence of desmosomal gene mutations, with conduction disease being the sole 'red flag' for the correct diagnosis. These findings may justify the need to exclude $L M N A$ mutations in patients with suspected arrhythmogenic cardiomyopathy, particularly when conduction disease is present.

This study confirms the malignant nature of laminopathies in terms of ventricular arrhythmias and progression to advanced heart failure. In our series sustained SVTs during the follow-up were more frequent in patients without neuromuscular involvement (23\% vs $7 \%$ ) with just 1 EDMD patient experiencing SVT. The low incidence of events in patients with skeletal myopathy differs from previous reports. Van Rijsingen et al. [10] reported that in patients with $L M N A$ mutations, the diagnosis of muscular dystrophy or a positive family history of muscular dystrophy was not associated with a different incidence of ventricular arrhythmias. The rate of SVT reported by the Authors was of $17 \%$ in EDMD patients and 19\% in non-EDMD patients. More than $20 \%$ of the whole population of this study required cardiac transplantation during follow-up and this is consistent with previous reports. Hasselberg et al. [11] reported that 19\% of genotyped LMNA patients underwent heart transplantation during a follow up of 8 years (median age 46 years). The need for heart transplantation in our series was independent from the involvement of the skeletal muscle and occurred at a median age of 45 years. Differently from what observed for other clinical events, neuromuscular involvement did not lead to an anticipation in the timeline and heart transplant was performed at a similar age in the two groups (median 43 vs 46 years).

In this series, patients with a neuromuscular presentation had a linear predictable progression over time (Fig. 7). Specifically, skeletal myopathy developed first, followed by arrhythmias (A-V block, sick sinus syndrome and AF in various combinations) and eventually, cardiomyopathy. This pattern of progression was not observed in the other patients.

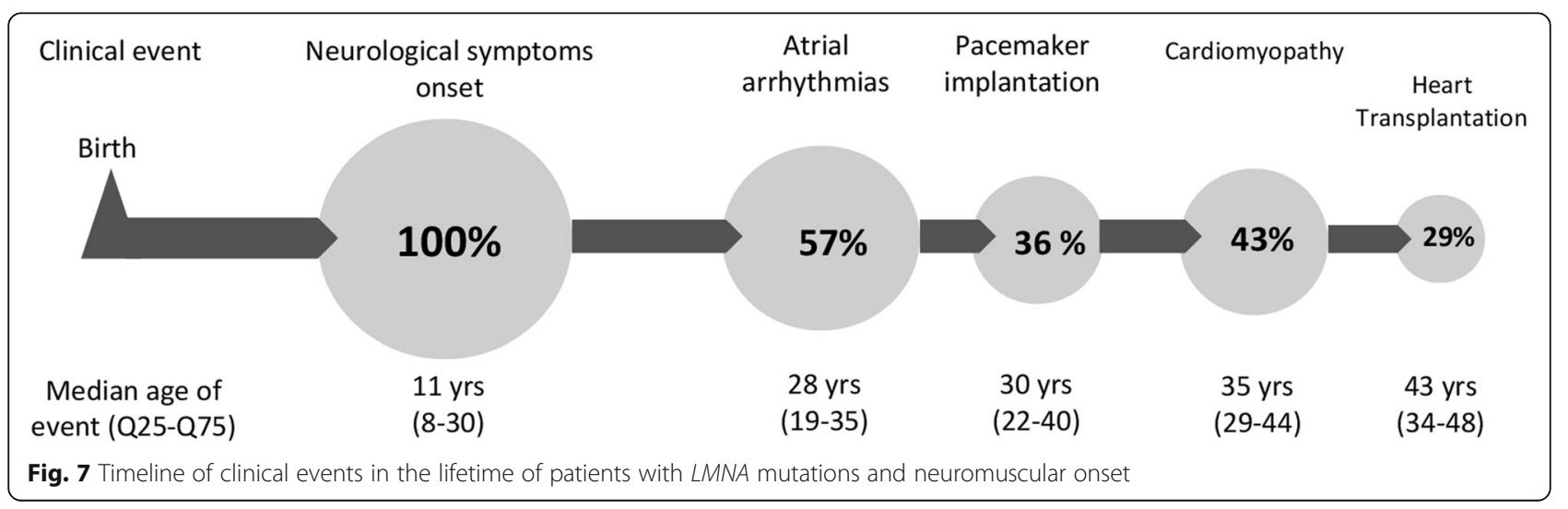


The cardiac involvement in X-linked EDMD patients of our study, compared to patients with LMNA mutations and a neurological onset, was characterized by higher burden of supraventricular tachyarrhyrhmias and bradyarrhythmias that occurred at a younger age and a lower frequency of cardiomyopathy.

\section{Limitations}

Referral bias cannot be excluded since this is a study from a single tertiary centre with a cardiac transplant program and expertise for management of complex ventricular tachycardias.

\section{Conclusion}

In patients with laminopathy, the type of disease onset was a marker for a different natural history. Specifically, patients with neuromuscular presentation had an earlier cardiac involvement, characterized by a linear and progressive evolution from rhythm disorders (AF and/or A-V block) to cardiomyopathy. Prevalence of AF and A$\mathrm{V}$ block was similar, regardless of clinical onset, whereas sinus node dysfunction was more frequent in EDMD patients. Patients with neuromuscular onset had a lower prevalence of cardiomyopathy and ventricular arrhythmias, but a similar prevalence of heart transplantation at a similar age.

\section{Abbreviations}

AF: Atrial fibrillation/flutter; AVB: Atrio-ventricular block; CK: Creatine kinase; CRT-D: Implantable cardiac resynchronization therapy defibrillator;

DCM: Dilated cardiomyopathy; EDMD: Emery Dreifuss muscular dystrophy; EMG: Electromyography; HNDC: Hypokinetic non dilated cardiomyopathy; HT: Heart transplantation; ICD: Implantable cardioverter defibrillator; IQR: Interquartile range; LV: Left ventricle; LVEF: Left ventricle ejection fraction; NYHA: New York Heart Association; PM: Pacemaker; SVT: Sustained ventricular tachyarrhythmias

\section{Acknowledgements}

None.

\section{Authors' contributions}

$E B, R D$ and $C R$ wrote the initial manuscript. $E B, C R, M B, G B, R D$ and $M L$ provided critical discussion of research. All Authors participated in clinical data collection, read and approved the final manuscript.

\section{Funding}

This work was supported by the University of Bologna and "Fondazione Luisa Fanti Melloni".

\section{Availability of data and materials}

The datasets used and analysed during the current study are available from the corresponding Author on reasonable request.

\section{Ethics approval and consent to participate}

Informed consent was obtained from patients for collection of anonymised clinical data.

\section{Consent for publication}

All participants gave consent for publication of anonymised individual personal data.

\section{Competing interests}

The authors declare that they have no competing interests.

\section{Author details}

'Cardiology Unit, Cardio-Thoracic-Vascular Department, Sant'Orsola-Malpighi Hospital, University of Bologna, Via G. Massarenti 9, 40138 Bologna, Italy. ${ }^{2}$ Cardiology Division, Department of Biomedical, Metabolic and Neural Sciences, University of Modena and Reggio Emilia, Policlinico di Modena, Modena, Italy. ${ }^{3}$ University College London Institute for Cardiovascular Science and Barts Heart Centre, St. Bartholomew's Hospital, London, UK. ${ }^{4}$ Neurology Unit, Sant'Orsola-Malpighi University Hospital, Bologna, Italy. ${ }^{5}$ Italian National Research Council (CNR), Institute of Molecular Genetics IGM Bologna, Bologna, Italy.

Received: 21 May 2019 Accepted: 29 October 2019

Published online: 19 November 2019

\section{References}

1. Mounkes L, Kozlov S, Burke B, Stewart CL. The laminopathies: nuclear structure meets disease. Curr Opin Genet Dev. 2003;13:223-30.

2. Shackleton $\mathrm{S}$, et al. LMNA, encoding Lamin $\mathrm{a} / \mathrm{C}$, is mutated in partial lipodystrophy. Nat Genet. 2000;24:153-6.

3. Boriani $\mathrm{G}$, et al. Clinical relevance of atrial fibrillation/flutter, stroke, pacemaker implant, and heart failure in Emery-Dreifuss muscular dystrophy: a long-term longitudinal study. Stroke. 2003;34:901-8.

4. Eriksson $\mathrm{M}$, et al. Recurrent de novo point mutations in Lamin a cause Hutchinson-Gilford progeria syndrome. Nature. 2003;423:293-8.

5. Bonne $\mathrm{G}$, et al. Mutations in the gene encoding Lamin a/C cause autosomal dominant Emery-Dreifuss muscular dystrophy. Nat Genet. 1999;21:285-8.

6. Arbustini $E$, et al. Autosomal dominant dilated cardiomyopathy with atrioventricular block: a Lamin a/C defect-related disease. J Am Coll Cardiol. 2002;39:981-90.

7. Kumar S, et al. Long-term arrhythmic and nonarrhythmic outcomes of Lamin a/C mutation carriers. J Am Coll Cardiol. 2016;68:2299-307.

8. Pasotti $\mathrm{M}$, et al. Long-term outcome and risk stratification in dilated cardiolaminopathies. J Am Coll Cardiol. 2008;52:1250-60.

9. Taylor MR, et al. Natural history of dilated cardiomyopathy due to Lamin a/ C gene mutations. J Am Coll Cardiol. 2003;41:771-80.

10. van Rijsingen IA, et al. Risk factors for malignant ventricular arrhythmias in Lamin a/c mutation carriers a European cohort study. J Am Coll Cardiol. 2012;59:493-500.

11. Hasselberg NE, et al. Lamin a/C cardiomyopathy: young onset, high penetrance, and frequent need for heart transplantation. Eur Heart J. 2018; 39:853-60.

12. Captur G, et al. Lamin and the heart. Heart. 2018;104:468-79.

13. Wahbi $K$, et al. Development and validation of a new risk prediction score for life-threatening ventricular Tachyarrhythmias in Laminopathies. Circulation. 2019;140:293-302.

14. Elliott P, et al. Classification of the cardiomyopathies: a position statement from the European Society of Cardiology working group on myocardial and pericardial diseases. Eur Heart J. 2008:29:270-6.

15. Pinto YM, et al. Proposal for a revised definition of dilated cardiomyopathy, hypokinetic non-dilated cardiomyopathy, and its implications for clinical practice: a position statement of the ESC working group on myocardial and pericardial diseases. Eur Heart J. 2016;37:1850-8.

16. Benedetti $\mathrm{S}$, et al. Phenotypic clustering of Lamin a/C mutations in neuromuscular patients. Neurology. 2007;69:1285-92.

17. Bonne $\mathrm{G}$, et al. Clinical and molecular genetic spectrum of autosomal dominant Emery-Dreifuss muscular dystrophy due to mutations of the Lamin a/C gene. Ann Neurol. 2000;48:170-80.

18. Brodsky GL, et al. Lamin a/C gene mutation associated with dilated cardiomyopathy with variable skeletal muscle involvement. Circulation. 2000;101:473-6.

19. Quarta G, et al. Mutations in the Lamin a/C gene mimic arrhythmogenic right ventricular cardiomyopathy. Eur Heart J. 2012;33:1128-36.

\section{Publisher's Note}

Springer Nature remains neutral with regard to jurisdictional claims in published maps and institutional affiliations. 